\title{
Numerical study of flow pattern modulation in a vertical phase separation condenser tube
}

\author{
SUN DongLiang ${ }^{1}$, XU JinLiang $^{1 *}$, CHEN QiCheng ${ }^{2} \&$ CAO Zhen ${ }^{2}$ \\ ${ }^{1}$ State Key Laboratory of Alternate Electrical Power System with Renewable Energy Sources, North China Electric Power University, Beijing \\ 102206, China; \\ ${ }^{2}$ Beijing Key Laboratory of Multi-Phase flow and Heat Transfer of Low-Grade Energy, North China Electric Power University, Beijing 102206, \\ China
}

Received October 18, 2012; accepted January 24, 2013

\begin{abstract}
The passive phase separation concept was proposed to modulate flow patterns for heat transfer enhancement. By the flow pattern modulation, the gas tends to be near the wall and the liquid tends to be in the tube core. Experiment has been performed to verify the fresh idea and the flow pattern modulation mechanism was analyzed qualitatively. This paper focuses on the numerical simulation of the bubble dynamics for a single bubble in the vertical phase separation condenser tube to quantitatively explore the flow pattern mechanism, based on a multiscale grid system and the volume-of-fluid (VOF) method. It is found that: (1) the modulated liquid film thickness can be decreased by $70 \%$ compared to that in the bare tube region; (2) the modulated bubble traveling velocity can be doubled, causing the increased liquid velocity and velocity gradient in the annular region to weaken the fluid boundary layer; (3) the significantly increased bubble traveling velocity in the annular region promotes the mass and momentum exchange between the annular region and the core region, and yields the self-sustained pulsating flow in the core region. The above three factors are benefit for the performance improvement of the heat transfer facilities.
\end{abstract}

phase separation, flow pattern modulation, mesh cylinder, condensation heat transfer, numerical simulation

Citation: $\quad$ Sun D L, Xu J L, Chen Q C, et al. Numerical study of flow pattern modulation in a vertical phase separation condenser tube. Chin Sci Bull, 2013, 58: 1592-1598, doi: 10.1007/s11434-013-5734-1

The low grade energy utilization has become a hot area due to the energy shortage and environment problems worldwide. The Organic Rankine Cycle (ORC) is one of the solutions to recover low grade energy (waste heat, solar energy and geothermal energy etc.) [1]. The major problem of ORC is the low thermal efficiency operating at low temperatures. The effective strategy for ORC performance improvement is to decrease the exergy loss for each component and whole system to a maximum degree. For the evaporator and condenser involved in ORC, the temperature difference across the two sides shall be decreased to reduce the exergy loss, yielding the improved heat transfer performance. Usually, the condensation heat transfer coefficient with organic fluid is less than $1 \mathrm{~kW} /\left(\mathrm{m}^{2} \mathrm{~K}\right)$ [2], which is on the same magni-

*Corresponding author (email: xjl@ncepu.edu.cn) tude of that for cooling water flowing in the shell side of the condenser. In other words, the thermal resistances at inside and outside of the tube are almost the same. Thus the enhancement of condensation heat transfer in the tube could significantly improve the condenser performance. With the multi-constraints of the increase of the utilization efficiency for low grade energy, decrease of the exergy loss and reduction of the fabrication cost, new principle and method to enhance the phase change heat transfer shall be developed.

The conventional heat transfer enhancement techniques involve the fluid disturbance and the delayed fluid boundary layer development. A micro-grooved tube [3-6] is one of the examples belonging to these techniques. Usually, gas populates in the tube core and liquid populates near the tube wall, yielding a larger thermal resistance due to the thick liquid thickness near the wall. The phase distribution and 
heat transfer are not coordinated to deteriorate the heat transfer. Therefore, the key scientific issue of the multiphase heat transfer enhancement is to coordinate the phase distribution and heat transfer. Lips and Meyer [7] experimentally studied the convective condensation heat transfer in an inclined tube with R134a as the working fluid. It is demonstrated that the phase distribution has significant effect on the heat transfer.

A new phase separation condenser tube was proposed using the passive phase separation concept to modulate flow patterns by our research group recently $[8,9]$. The tube cross section is divided into an annular region and a core region by suspending a mesh pore cylinder in the tube. Due to the surface tension effect, the gas bubbles are modulated to flow in the annular region and liquid is flowing in the core region. Such phase distribution is totally inverse to that in a conventional tube. The flow pattern and heat transfer are coordinated. Experiments have been performed to verify the flow pattern modulation by the passive phase separation concept. The flow pattern modulation mechanism has been analyzed qualitatively.

In order to thoroughly explore the mechanisms of the flow pattern modulation, this paper focuses on the numerical simulation of the bubble dynamics for a single bubble flowing in the vertical phase separation condenser tube. The particle-trajectory model [10], two-fluid model [11] and interface-tracking model are the main numerical models for two-phase flow and heat transfer problems. Among them, the interface-tracking model could accurately and completely acquire the interface information. The volume-of-fluid (VOF) method [12] and level set (LS) method [13] are the two major methods for the interface tracking, which are very popular and widely used. Currently, FLUENT uses the VOF method to capture the interface, having wide applications [14]. In this paper, we use the VOF method imbedded in FLUENT and the multiscale grid system to investigate the bubble dynamics for a single bubble flowing in both the bare tube region and the modulated flow region. The liquid film thicknesses, bubble traveling velocity and velocity field are obtained to analyze the mechanism of the flow pattern modulation by the passive phase separation concept.

\section{Condenser tube configuration and grid system}

Figure 1 shows the vertical phase separation condenser tube. The tube has a bare tube region and a modulated flow region, separated by the side surface of a suspended mesh cylinder made of a single layer of metal porous surface. The two regions have the lengths of 500 and $350 \mathrm{~mm}$ respectively. In the modulated flow region, the tube cross section is divided into an annular region and a core region, interfaced by the mesh cylinder surface. The tube has a diameter of $15.7 \mathrm{~mm}$. The modulated region has an annular gap of $2.6 \mathrm{~mm}$ and a diameter of $10.36 \mathrm{~mm}$ for the core region.

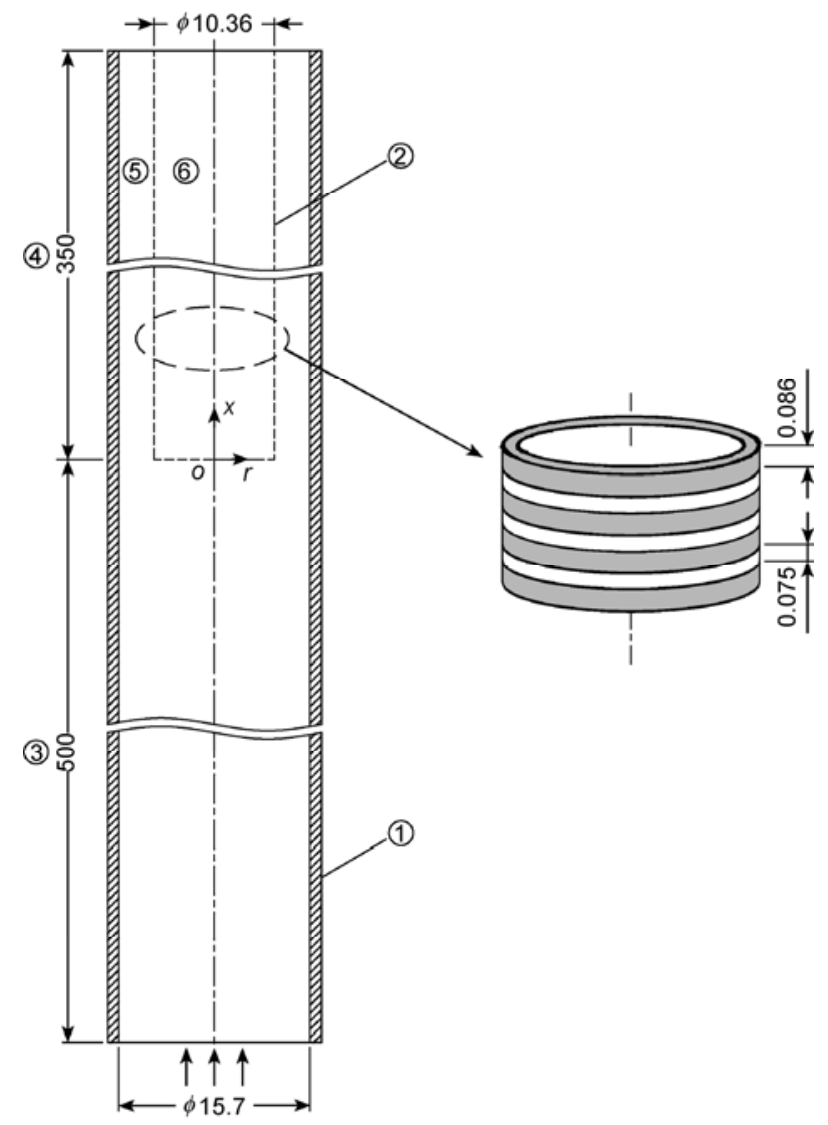

Figure 1 Vertical phase separation condenser tube (unit: $\mathrm{mm}$ ). (1) Tube wall; (2) mesh cylinder; (3) bare tube region; (4) modulated flow region; (5) annular region; (6) core region.

The metal wire thickness is $0.086 \mathrm{~mm}$ and the mesh pore has a width of $0.075 \mathrm{~mm}$.

We perform the numerical simulation of the flow pattern modulation process for a single bubble with vertical upflows. Due to the configuration symmetry, the two-dimensional axial-symmetric coordinate $(r-x)$ system is selected to perform the numerical simulations. Due to the inserted mesh cylinder, three types of geometric scales exist: (1) the bare tube region and the core region have the conventional macroscale; (2) the annular gap has miniature scale with a couple of millimeters; (3) the mesh pores have micron scale. For such a complicated multiscale geometry system, the grid number will be significantly large if one uses the uniform grid system. The grid number is 50 million with a uniform grid system. The computation time is estimated to be several years for the unsteady two-phase flow problem even though the parallel computation technique is used, making impossible to fulfill the numerical simulation. Here we develop a grid system with different grid scales, matching different geometric sizes for each part of the condenser tube. The bare tube and core regions possess the average grid sizes of $0.5 \mathrm{~mm}$. The annular gap has the grid size of $0.1 \mathrm{~mm}$, and the mesh pore has the grid size of $10 \mu \mathrm{m}$. The different grid sizes are linked with each other by adapting 
the transition pattern $5: 1$. Figure 2 illustrates the final used multiscale grid system. The grid number is 300000 , which is only $0.6 \%$ of that for a uniform grid-scale system.

In the VOF method, a volume fraction function $\alpha$ is defined to denote whether a space is occupied by the gas or liquid. When $\alpha=1$, the space is occupied by the gas. When $\alpha=0$, the space is occupied by the liquid. When $0<\alpha<1$, the space contains both the gas and liquid, where by the definition an interface exists. In order to accurately capture the bubble interface, a dynamic gradient adaption technique is used near the bubble interface, with two levels of grid refinement. As shown in Figure 3, the grid near the interface is refined by sixteen times compared to that far away from the interface.

\section{Governing equations and solution strategy}

We perform the numerical simulation of the flow pattern modulation process for a single bubble flowing in the continuous liquid phase. Air is the gas phase and water is the liquid phase. The initial bubble shape and size and related boundary conditions are shown in Figure 4 . The fully de- veloped velocity distribution at the inlet is given as

$$
u_{\text {in }}=2 \bar{u}_{\text {in }}\left[1-\left(\frac{r}{0.00785}\right)^{2}\right] \text {, }
$$

where $\bar{u}_{\text {in }}$ is the average velocity at the inlet, which is $0.1 \mathrm{~m} / \mathrm{s}$ in this study. The studied problem is an unsteady incompressible laminar two-phase flow problem. The whole computation domain only needs a set of governing equations when VOF method is used. The details are as follows.

The advection equation of the volume fraction is

$$
\frac{\partial \alpha}{\partial t}+\nabla \cdot(\vec{v} \alpha)=0
$$

The continuity equation is

$$
\nabla \cdot \vec{v}=0
$$

The momentum equation is

$$
\frac{\partial}{\partial t}(\rho \vec{v})+\nabla \cdot(\rho \vec{v} \vec{v})=-\nabla p+\nabla \cdot\left[\mu\left(\nabla \vec{v}+\nabla \vec{v}^{\mathrm{T}}\right)\right]+\rho \vec{g}+F_{\mathrm{vol}},
$$

(a)

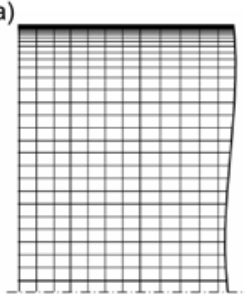

Tube wall

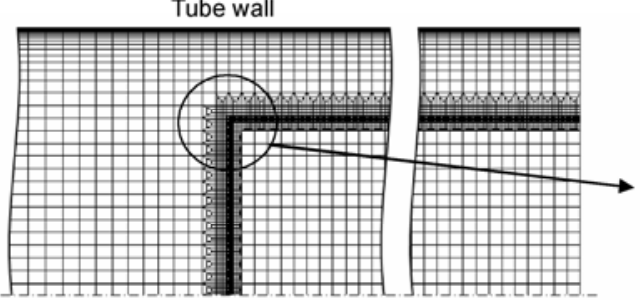

Symmetric axis (b)

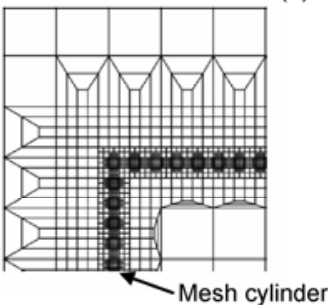

Figure 2 Multiscale grid system of vertical phase separation condenser tube. (a) Grid system; (b) local enlarging grid.

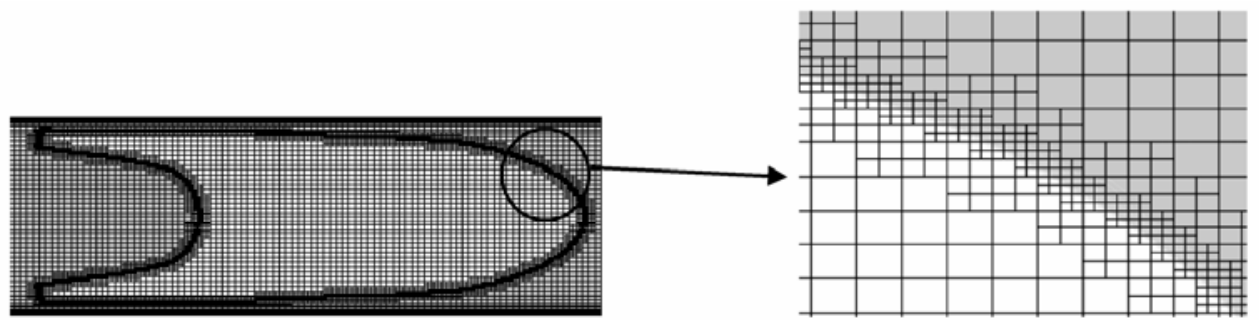

(a)

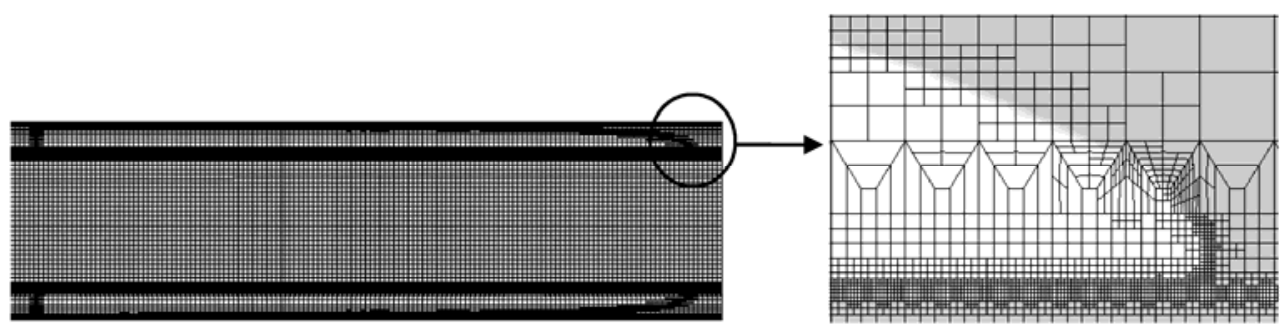

(b)

Figure 3 Grid near bubble interface in bare tube region and modulated flow region. (a) Bare tube region; (b) modulated flow region. 


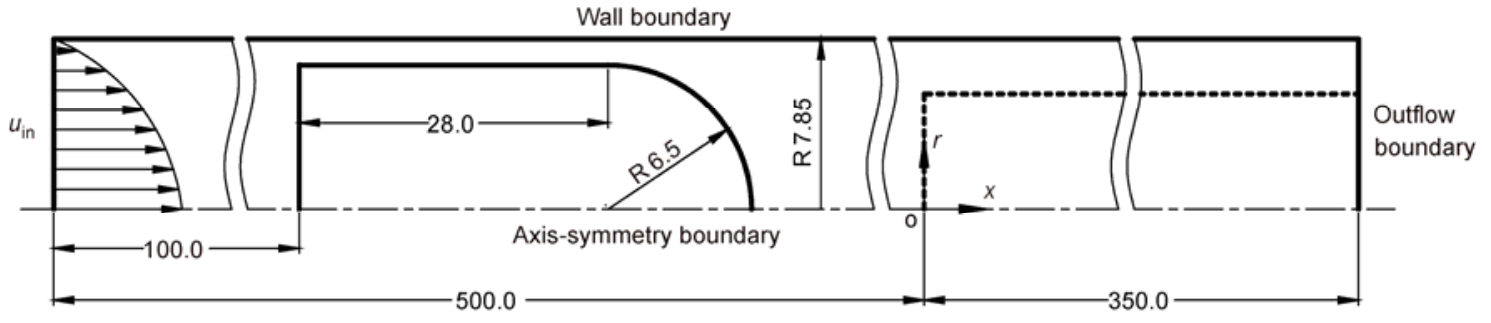

Figure 4 Initial bubble shape and boundary conditions (unit: $\mathrm{mm}$ ).

in which

$$
\begin{aligned}
& \rho=\rho_{\mathrm{g}} \alpha+\rho_{\mathrm{l}}(1-\alpha), \\
& \mu=\mu_{\mathrm{g}} \alpha+\mu_{\mathrm{g}}(1-\alpha) .
\end{aligned}
$$

The CSF(continuum surface force) model [15] is used to convert the surface force due to the surface tension effect into the body force.

$$
F_{\text {vol }}=\sigma \frac{\alpha \rho_{\mathrm{g}} \kappa_{\mathrm{g}} \nabla \alpha+(1-\alpha) \rho_{\mathrm{l}} \kappa_{1} \nabla(1-\alpha)}{0.5\left(\rho_{\mathrm{g}}+\rho_{1}\right)},
$$

in which

$$
\kappa_{\mathrm{g}}=-\kappa_{l}=-\nabla \cdot\left(\frac{\nabla \alpha}{|\nabla \alpha|}\right)
$$

In eqs. (2)-(8), $t$ is time, $\vec{v}$ is velocity vector, $\vec{g}$ is gravity acceleration, $p$ is pressure, $\rho$ is density, $\mu$ is dynamic viscosity, $\sigma$ is surface tension coefficient, $\kappa$ is interface curvature and the subscripts $g$ and 1 denote, respectively, the gas and liquid phases.

We apply the PLIC method to solve the advection equa- tion of the volume fraction eq. (2). The SIMPLE algorithm is used to couple the pressure and velocity, thus the continuity equation eq. (3) and momentum equation eq. (4) are solved. The MUSCL scheme is adopted for the convection term in the momentum equation. The simulations are run on a work station having two 8-core CPUs (2.4 GHz each) and $24 \mathrm{~GB}$ of RAM. The computation needs ten days for each run.

\section{Results and discussion}

Figure 5 shows the transient slug bubble shapes in both the bare tube region and the modulated flow region at different time. When the slug bubble attaches the mesh cylinder surface, the penetrating of a bubble from a larger space into a small space needs a larger surface energy due to the surface tension effect. Thus the slug bubble cannot completely breakthrough the mesh pores, forcing the bubble flowing in the annular region to form the elongated-ring-slug-bubble. Generally, the annular region occupies the elongated-ringslug-bubble segmented by a set of liquid plugs, and the core region is completely full of liquid.

Figure 6 shows the liquid film thickness in both the bare
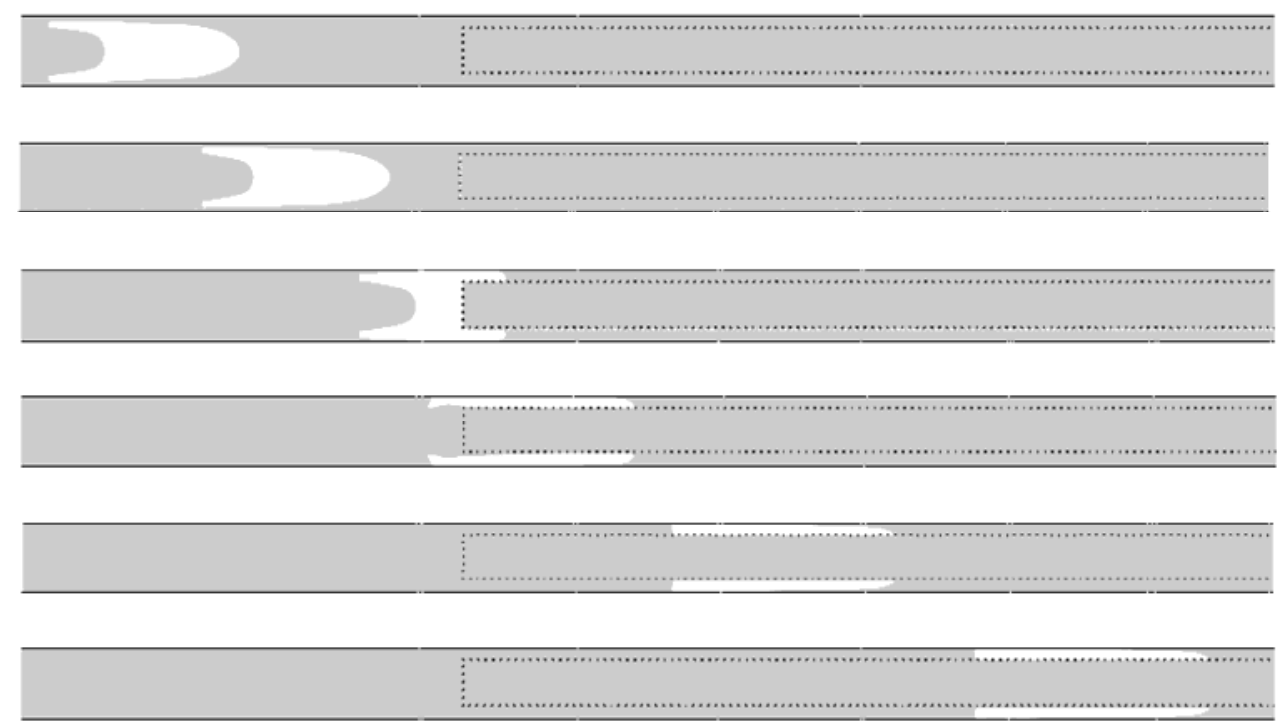

Figure 5 Flow pattern modulation process of mesh cylinder for a single slug bubble. $x_{\mathrm{s}}=-100 \mathrm{~mm}, x_{\mathrm{e}}=180 \mathrm{~mm}, x_{\mathrm{s}}$ and $x_{\mathrm{e}}$ represent the starting and ending locations of the visualization area along the flow direction. 


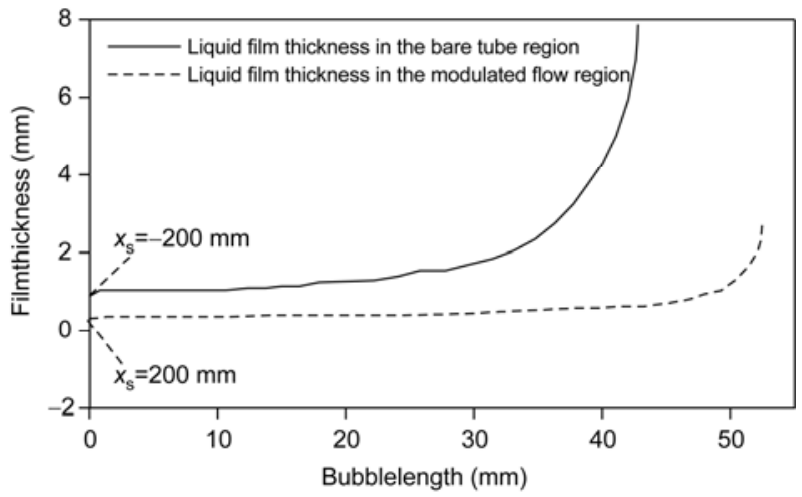

Figure 6 Liquid film thickness in both the bare tube region and the modulated flow region.

tube region and the modulated flow region. The average liquid film thickness is $1.82 \mathrm{~mm}$ in the bare tube region and $0.55 \mathrm{~mm}$ in the modulated flow region. The liquid film thickness is modulated to be decreased by $70 \%$ compared to that in the bare tube region. During the phase change heat transfer process, the wall heat flux at the liquid film can be written in the following form, assuming the constant wall temperature, constant saturation temperature in vapor phase and linear temperature distribution within the liquid film.

$$
q_{\mathrm{w}}=\lambda_{\mathrm{I}} \frac{T_{\text {Sat }}-T_{\mathrm{w}}}{h},
$$

where $\lambda_{1}$ is liquid thermal conductivity, $T_{\text {Sat }}$ is saturation temperature, $T_{\mathrm{w}}$ is wall temperature, $h$ is liquid film thickness and $q_{\mathrm{w}}$ is wall heat flux. The liquid film thickness directly determines the wall heat flux. Due to the liquid film thickness decreased by $70 \%$ after the flow pattern modulation, the wall heat flux at the liquid film is expected to be 3.3 times higher than that in the bare tube region.

Figure 7 shows the slug bubble velocities versus the axial flow direction in both the bare tube region and modulated flow region. The slug bubble velocity is averaged to be 0.29 $\mathrm{m} / \mathrm{s}$ in the bare tube region. When such a bubble attaches

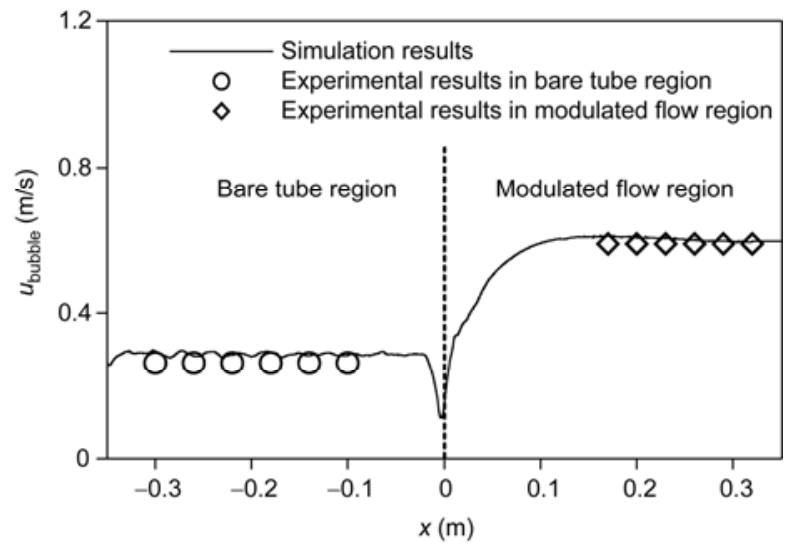

Figure 7 Slug bubble rising velocities at different axial flow positions.

the side surface of the mesh cylinder, the bubble velocity is instantly decreased to $0.11 \mathrm{~m} / \mathrm{s}$ due to the obstacle of the mesh cylinder. The bubble velocities are sharply increased and then keep stabilized when the bubble fully enters the annular region. The stabilized slug bubble velocity is $0.6 \mathrm{~m} / \mathrm{s}$ in the modulated flow region. It is seen from Figure 7 that the computed slug bubble velocities match the measured values well, verifying that the present multiscale grid system and numerical method are accurate and feasible.

The slug bubble velocities are significantly increased from $0.29 \mathrm{~m} / \mathrm{s}$ in the bare tube region to $0.60 \mathrm{~m} / \mathrm{s}$ in the modulated flow region, which are doubled by the flow pattern modulation. The increased ring-slug bubble velocity causes the faster liquid movement in the annular region. Figure 8 illustrates the axial velocity distribution versus the radial direction at the cross section $\mathrm{AB}$ in the bare tube region and cross section $\mathrm{CD}$ in the modulated flow region. Both cross sections $\mathrm{AB}$ and $\mathrm{CD}$ are ahead of the bubble front. It is seen from Figure 8 that both the velocity and velocity gradient near the wall at the cross section CD by the flow pattern modulation are larger than those at the cross section $\mathrm{AB}$ in the bare tube section. The increased velocity and velocity gradient near the wall reduces the fluid
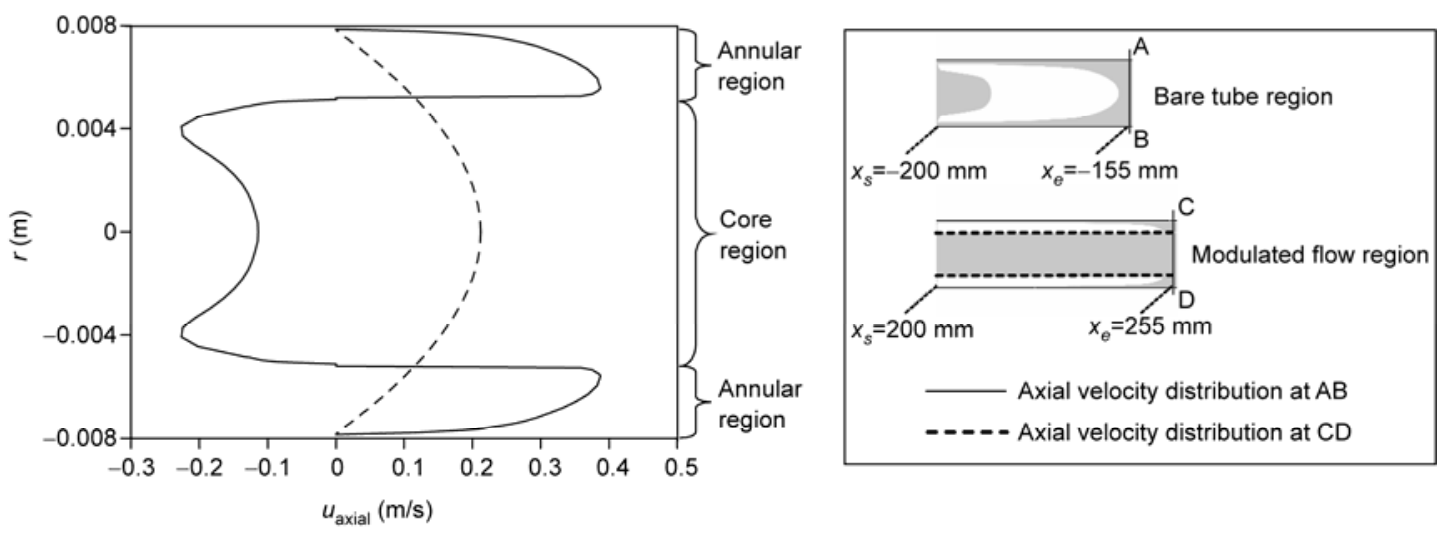

Figure 8 Axial velocity distributions at different cross sections. 
boundary layer, which is benefit for the heat transfer enhancement.

The significantly increased ring-slug bubble velocity in the annular region enhances the mass and momentum exchange between the annular region and the core region across the mesh pores. As shown in Figure 9, the liquid ahead of the ring-slug bubble front flows from the annular region towards the core region. On the other hand, liquids at the ring-slug bubble tail are periodically oscillating and finally return to the annular region. The mass and momentum exchange across the mesh pores results in the self-sustained pulsating flow in the core region. Liquids in the core region behave the periodic upflow and downflow phenomenon (see Figure 10). The mass and momentum exchange across the mesh pores and the self-sustained pulsating flow in the core region are benefit for the liquid mixing between the near wall region and the core region, accounting for the overall performance improvement of the heat transfer facilities.

\section{Conclusions}

This paper numerically investigates the flow pattern modulation process in a newly proposed condenser tube for ver- tical upflows based on the multiscale grid system and VOF method. The liquid film thickness, bubble traveling velocity and velocity field are studied in both the bare tube region and modulated flow region. The following conclusions can be drawn.

(1) The average liquid film thickness is decreased from $1.82 \mathrm{~mm}$ in the bare tube region to $0.55 \mathrm{~mm}$ in the modulated flow region, accounting for the reduction of $70 \%$ by the modulated flow. The wall heat flux at the liquid film in the modulated flow region is expected to be 3.3 times higher than that in the bare tube region.

(2) The slug bubble velocity is increased from $0.29 \mathrm{~m} / \mathrm{s}$ in the bare tube region to $0.6 \mathrm{~m} / \mathrm{s}$ in the modulated flow region, which is doubled by the modulated flow.

(3) The increased bubble velocity in the annular region raises the liquid velocity and velocity gradient in the annular region, which are benefit for the performance improvement of the heat transfer facilities.

(4) The mass and momentum exchange across the metal mesh pores and the self-sustained pulsating flow in the core region are useful for the liquid mixing between the near wall region and the core region, helping the performance improvement of the heat transfer facilities.

We see from the above analyses that the modulated flow

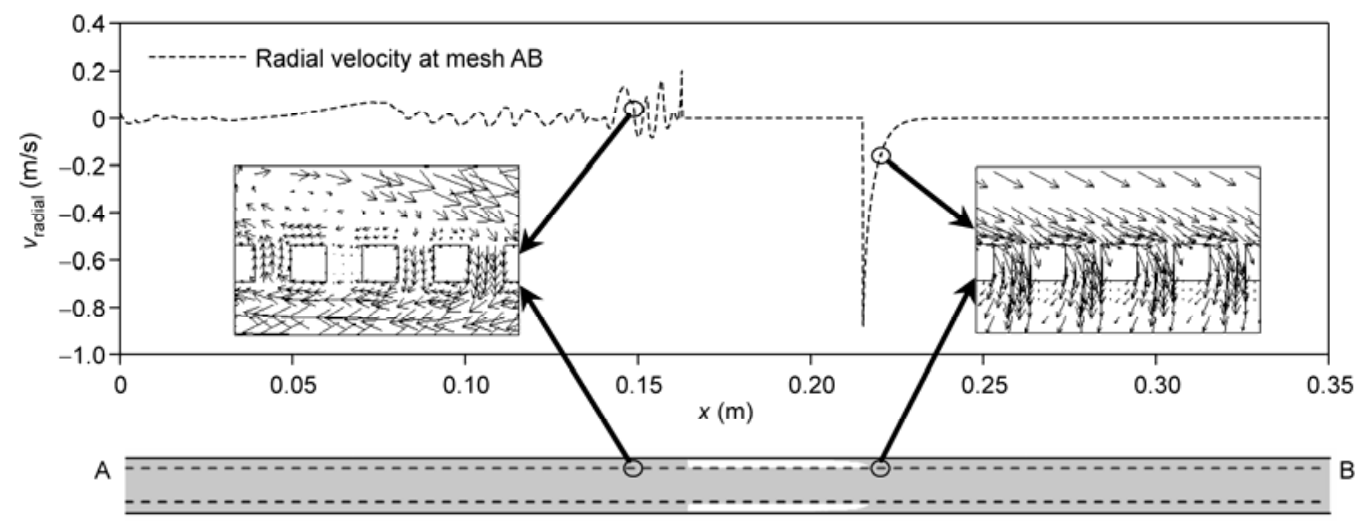

Figure 9 Fluid exchange between the annular region and the core region.

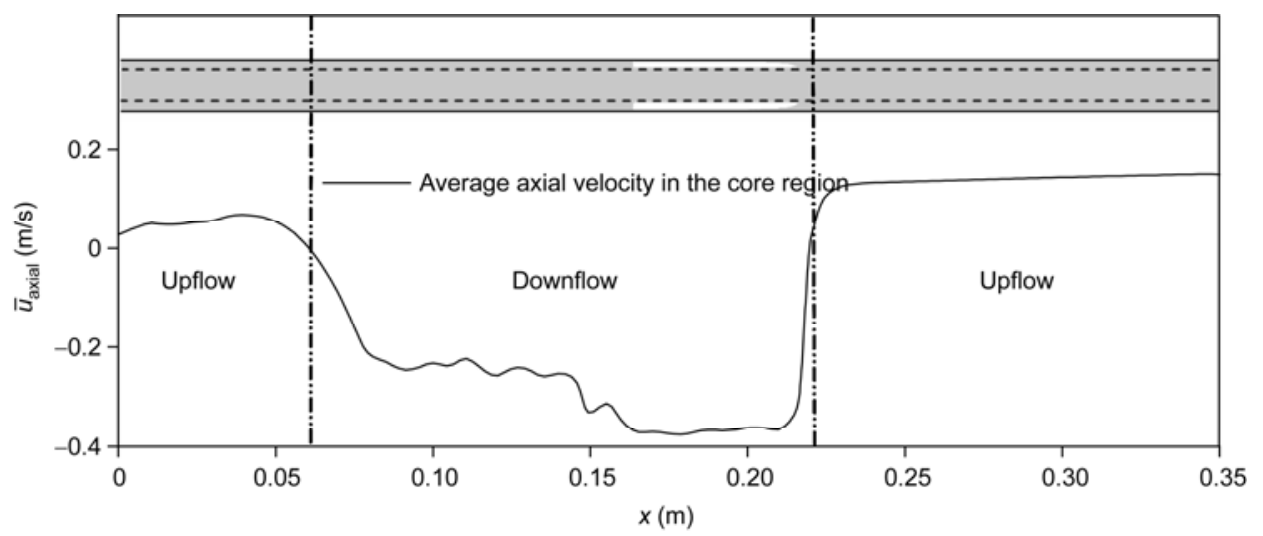

Figure 10 Self-sustained pulsating flow in the core region. 
pattern ensures not only the thin liquid film flow mode with "the gas near the wall and liquid in the core", but also the improved velocity field. All these factors are benefit for the performance improvement of the heat transfer facilities.

This work was supported by the Young Scientists Fund of the National Natural Science Foundation of China (51106049), the National Basic Research Program of China (2011CB710703), the Natural Science Foundation of China of International Cooperation Project (51210011), the Beijing Natural Science Foundation (3112022), the Hebei Natural Science Foundation (E2011502057) and the Fundamental Research Funds for the Central Universities (12MS44)

1 Chen Q C, Xu J L, Chen H X. A new design method for Organic Rankine Cycles with constraint of inlet and outlet heat carrier fluid temperatures coupling with heat source. Appl Energ, 2012, 98: 562573

2 Laohalertdecha S, Wongwises S. The effects of corrugation pitch on the condensation heat transfer coefficient and pressure drop of R-134a inside horizontal corrugated tube. Int J Heat Mass Tran, 2010, 53: 2924-2931

3 Graham D M, Chato J C, Newell T A. Heat transfer and pressure drop during condensation of refrigerant 134a in an axially grooved tube. Int J Heat Mass Tran, 1999, 42: 1935-1944

4 Cavallini A, Del Col D, Doretti L, et al. Heat transfer and pressure drop during condensation of refrigerants inside horizontal enhanced. Int J Refrig, 2000, 23: 4-25

5 Miyara A, Otsubo Y. Condensation heat transfer of herringbone mi- cro fin tubes. Int J Therm Sci, 2002, 41: 639-645

6 Suriyan L, Somchai W. The effects of corrugation pitch on the condensation heat transfer coefficient and pressure drop of R134a inside horizontal corrugated tube. Int J Heat Mass Tran, 2010, 53: 29242931

7 Lips S, Meyer J P. Experimental study of convective condensation in an inclined smooth tube. Part I: Inclination effect on flow pattern and heat transfer coefficient. Int J Heat Mass Tran, 2012, 55: 395-404

8 Chen H X, Xu J L, Li Z J, et al. Flow pattern modulation in a horizontal tube by the passive phase separation concept. Int J Multiphas Flow, 2012, 45: 12-23

9 Chen H X, Xu J L, Wang W. An enhancement condensation pipe with inserted mesh cylinder (in Chinese). PRC Patent, CN102278904A, 2011-12-14

10 Luo K, Fan J R, Cen K F. Transitional phenomenon of particle dispersion in gas-solid two-phase flows. Chin Sci Bull, 2007, 52: 408417

11 Morales-Ruiz S, Rigola J, Rodriguez I, et al. Numerical resolution of the liquid-vapour two-phase flow by means of the two-fluid model and a pressure based method. Int J Multiphas Flow, 2012, 43: 118130

12 Hirt C W, Nichols B D. Volume of fluid (VOF) method for the dynamics of free boundary. J Comput Phys, 1981, 39: 201-225

13 Yan Z L, Xie W J, Geng D L, et al. The ninth-mode sectorial oscillation of acoustically levitated drops. Chin Sci Bull, 2011, 56: 32843288

14 Araujo J D P, Miranda J M, Pinto A M F R, et al. Wide-ranging survey on the laminar flow of individual Taylor bubbles rising through stagnant Newtonian liquids. Int J Multiphas Flow, 2012, 43: 131-148

15 Brackbill J U, Kothe D B, Zemach C. A continuum method for modeling surface tension. J Comput Phys, 1992, 100: 335-354

Open Access This article is distributed under the terms of the Creative Commons Attribution License which permits any use, distribution, and reproduction in any medium, provided the original author(s) and source are credited. 